\title{
Refresher-CME
}

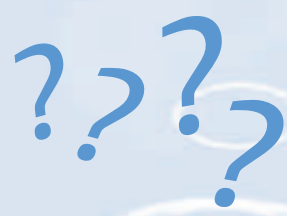

00

Die folgenden Fragen beziehen sich auf den vorangehenden Beitrag. Bitte schicken

Sie uns die entsprechenden Lösungsbuchstaben. Jeweils eine Antwort ist richtig.

Die Vergabe von CME-Punkten ist an die korrekte Beantwortung der Multiple-Choice-

Fragen gebunden.

Frage 1
Glomus-tympanicum-Tumore sind
am häufigsten lokalisiert

Frage 2

Welche Aussage bezüglich des Stapes ist richtig?

Frage 3

Welche Aussage bezüglich des ovalen Fensters ist falsch?

Frage 4

Welche Aussage/n ist/sind richtig?
A im Hypotympanon
B im Epitympanon
C auf der Paries mastoideus
D auf dem Promontorium
E auf der Paries caroticus

A Die Fußplatte des Stapes ist in der Fenestra cochleae lokalisiert.

B Die Fußplatte des Stapes ist in der Fenestra vestibuli lokalisiert.

C Die Fußplatte des Stapes ist im Umbo lokalisiert.

(D) Die Fußplatte des Stapes weist üblicherweise Dehiszenzen auf.

E Die Fußplatte des Stapes artikuliert mit dem Incus im Incudostapedialgelenk.

A Das ovale Fenster trennt das Mittelohr vom Labyrinth.

B Das ovale Fenster kann bei einer Otosklerose durch Plaques eingeengt sein.

C Der Processus lenticularis sitzt im ovalen Fenster.

(D) Die Fläche des ovalen Fensters beträgt ca. $3 \mathrm{~mm}^{2}$.

E Das ovale Fenster liegt kaudal der Prominentia canalis facialis.

(1. Im inneren Gehörgang liegt der N. facialis dorsal kranial.

2. Im inneren Gehörgang liegt der N. facialis dorsal kaudal.

3. Im inneren Gehörgang sind der N. cochlearis und die Pars superior des N. vestibularis V-förmig angeordnet.

4. Im inneren Gehörgang verlaufen der N. cochlearis und der N. facialis parallel.

5. Im inneren Gehörgang liegt der N. cochlearis ventral kaudal.

A Nur Aussage 5 ist richtig

B Nur Aussage 2 ist richtig

C Nur die Aussagen 1 und 4 sind richtig

D Nur die Aussagen 2 und 3 sind richtig

(E) Nur die Aussagen 2, 3 und 5 sind richtig 
Frage 5

Welche Aussage/n ist/sind richtig?

\section{Frage 6}

Welche Aussage zum N. facialis ist falsch?

Frage 7

Welche Aussage zum Trommelfell ist falsch?

Frage 8

Welche anatomischen Strukturen weisen keine Dehiszenzen auf?

Frage 9

Welche Aussage/n ist/sind falsch?

Frage 10

Welche Aussage/n ist/sind richtig?
1. Eine Parese des N. stapedius führt zur Hyperakusis.

2. Eine Parese des N. stapedius führt zur Hypakusis.

3. Ein Parese des N. tensor tympani führt zur Hypakusis.

4. Ein Parese des N. tensor tympani führt zur Hyperakusis.

5. Der N. stapedius entspringt dem N. facialis kaudal des Abgangs der Chorda tympani.

A Nur Aussage 5 ist richtig

B Nur die Aussagen 1 und 3 sind richtig

C Nur die Aussagen 2 und 4 sind richtig

(D) Nur die Aussagen 1, 3 und 5 sind richtig

E Nur die Aussagen 2, 4 und 5 sind richtig

A Das labyrinthäre Segment wird als $\mathrm{S}_{1}$-Segment bezeichnet.

B Das mastoidale Segment wird als $S_{3}$-Segment bezeichnet.

C Das tympanale Segment wird als $\mathrm{S}_{2}$-Segment bezeichnet.

D Der Fazialiskanal weist selten Dehiszenzen auf.

E Der Fazialisverlauf ist sehr variabel.

A Das Trommelfell wird in eine Pars tensa und Pars flaccida unterteilt.

B Die Pars tensa besteht aus drei Zellschichten.

C Das erworbene Cholesteatom geht überwiegend vom kaudalen Anteil des Trommelfells aus.

D Das erworbene Cholesteatom geht überwiegend von der Flaccida aus.

E Das Trommelfell wird in seiner Mitte durch den Hammergriff eingezogen.

A der Fazialiskanal

B der Carotiskanal

C der innere Gehörgang

D das Tegmen tympani

E der Kanal des anterioren Bogengangs

1. Das Crus commune wird vom anterioren und lateralen Bogengang gebildet.

2. Das Crus commune wird vom anterioren und posterioren Bogengang gebildet.

3. Das Labyrinth besteht aus Cochlea und Vestibularorgan.

4. Die retrofenestrale Otosklerose befällt das Labyrinth und kann zu Sklerosierungen führen.

5. Der Aquaeductus cochleae mündet an der Pyramidenhinterkante.

A Nur Aussage 1 ist falsch

B Nur Aussage 2 ist falsch

C Nur die Aussagen 2 und 4 sind falsch

D Nur die Aussagen 2 und 5 sind falsch

E Nur die Aussagen 1 und 5 sind falsch

1. Der Aquaeductus vestibuli ist ab einem Durchmesser von $1,5 \mathrm{~mm}$ pathologisch erweitert.

2. Der Aquaeductus vestibuli führt vom Vestibulum zur Pyramidenhinterkante.

3. Der Aquaeductus vestibuli beherbergt den Ductus perilymphaticus.

4. Der Aquaeductus vestibuli verläuft medial zum posterioren Bogengang.

5. Der Aquaeductus vestibuli führt zum Kleinhirnbrückenwinkel.

A Nur Aussage 1 ist richtig

B Nur die Aussagen 1,2 und 3 sind richtig

C Nur die Aussagen 1, 2 und 4 sind richtig

(D) Nur die Aussagen 1, 3 und 4 sind richtig

E Nur die Aussagen 1,2 und 5 sind richtig 